\title{
Extension of Ultrasound Fourier Slice Imaging Theory to Sectorial Acquisition
}

\author{
Miaomiao Zhang, Adrien Besson, Rafael E. Carrillo, François Varray, Hervé Liebgott, Jean-Philippe Thiran, \\ Denis Friboulet, and Olivier Bernard
}

\begin{abstract}
Ultrasound image reconstruction from the echoes received by an ultrasound probe after the transmission of diverging waves is an active area of research because of its capacity to insonify at ultra-high frame rate with large regions of interest using small phased arrays as the ones used in echocardiography. Current state-of-the-art techniques are based on the emission of diverging waves and the use of delay and sum strategies applied on the received signals to reconstruct the desired image (DW/DAS). Recently, we have introduced the concept of Ultrasound Fourier Slice Imaging (UFSI) theory for the reconstruction of ultrafast imaging for linear acquisition. In this study, we extend this theory to sectorial acquisition thanks to the introduction of an explicit and invertible spatial transform. Starting from a diverging wave, we show that the direct use of UFSI theory along with the application of the proposed spatial transform allows reconstructing the insonified medium in the conventional Cartesian space. Simulations and experiments reveal the capacity of this new approach in obtaining competitive quality of ultrafast imaging when compared with the current reference method.
\end{abstract}

Index Terms - Ultrafast ultrasound imaging, Diverging wave, Fourier beamforming.

\section{INTRODUCTION}

$\mathbf{U}$ LTRAFAST ultrasound imaging based on diverging wave faces an increasing interest in cardiac imaging due to its ability to perform high frame rate acquisition with large sector angles. In conventional ultrasound imaging, each reconstructed line of the B-mode image comes from the emission of one focused beam. In this context, the acquisition of one ultrasound image with a sector of $90^{\circ}$ and composed by 128 lines with an imaging depth of $10 \mathrm{~cm}$ can be performed with a frame rate around $60 \mathrm{~Hz}$. Contrary to this technique, ultrafast imaging based on diverging wave has the potential of acquiring the same type of sectorial image but with a frame rate more than 10 times faster.

The reference methods for ultrafast cardiac imaging are all spatial-based approaches where the desired image is obtained from a delay-and-sum (DAS) beamforming in the space domain [1], [2]. Recently, we have introduced the new concept of ultrasound Fourier slice imaging (UFSI) theory for the

O. Bernard, M. Zhang, F. Varray, H. Liebgott and D. Friboulet are with the University of Lyon, CREATIS, CNRS UMR5220, Inserm U630, INSA-Lyon, University of Lyon 1, Villeurbanne, France. E-mail: olivier.bernard@creatis.insa-lyon.fr

A. Besson, J.P. Thiran and R.E. Carrillo are with the Signal Processing Laboratory (LTS5), Ecole polytechnique fédérale de Lausanne (EPFL), Lausanne, Switzerland.

J.P. Thiran and R.E. Carrillo are also with the Department of Radiology, University Hospital Center (CHUV) and University of Lausanne (UNIL), Lausanne, Switzerland. reconstruction of ultrafast images for linear acquisition using plane wave [3]. In this study, we propose to extend the concept of UFSI to sectorial acquisition with the use of diverging wave in emission. In particular, we show that the application of UFSI theory for plane wave directly on the raw-data acquired through the use of a diverging wave in emission and following by a dedicated spatial transformation allow reconstructing a sectorial ultrasound image in the conventional cartesian space.

The paper is organized as follows. In Section II, the reconstructed scheme dedicated to sectorial acquisition is presented. In particular we show how Fourier slice imaging theory can be exploited to reconstruct an ultrasound images with diverging wave in emission. The quality of the obtained image is then investigated through both numerical simulation and experimental data in Section III. Comparisons with the existing techniques are also provided. Finally, a conclusion is given in Section IV.

\section{Methodology}

In this section, the general concept of UFSI for plane wave is first recalled in Sec.II-A. The novelty of the proposed work, i.e. the extension of the UFSI formalism to sectorial acquisition is then described in Sec.II-B.

\section{A. UFSI formalism for linear acquisition}

As shown in Fig. 1, the principle of UFSI theory can be summarized as follows. A 1-D linear array transducer located at $z=0$ is excited to generate a horizontal plane wave with direction $\mathbf{n}_{e}=(0,1)^{T}$ to insonify the medium. The same transducer is used to receive the back-scattered echoes $s\left(x_{i}, t\right)$. Different delays are then applied in reception in order to receive steered plane waves with varying angles $\xi$. It has been demonstrated in [3] that in such a case, the temporal Fourier transform of the sum of all the received signals $\left(s\left(x_{i}, t\right)\right.$ in Fig.1) corresponds to a radial line of angle $(\theta=\xi / 2)$ in the corresponding $\mathrm{k}$-space of the desired image. Changing the delays applied in the received signals, it is possible to radially and densely recover the Fourier space of the object and thus reconstruct an ultrasound image with high frame rate.

\section{B. Extension of UFSI to sectorial acquisition}

The key idea introduced in this work is to establish an equivalence in terms of traveling time when either a diverging wave or a plane-wave are used in emission. Indeed, such a relation would allow the reconstruction of a sectorial image 


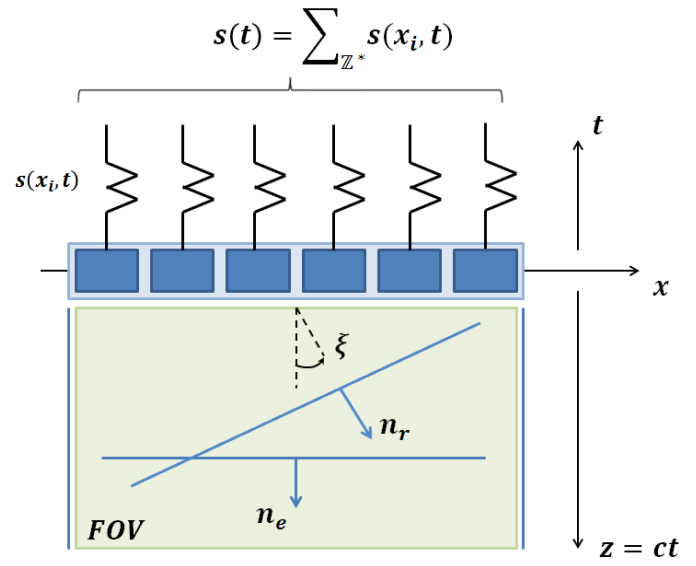

Fig. 1. Illustration of image formation using UFSI scheme.

from a diverging wave using the same formalism as the one derived for linear acquisition.

Let's define a probe located at $z=0$, which center at $x=$ 0 and where each transducer element is positioned at $(x, 0)$. Ultrafast techniques for sectorial acquisition are based on the emission of diverging wave [1], [2]. Such a wave is obtained through the excitation of a virtual source located behind the probe and placed at $\left(0, z_{n}\right)$, where $z_{n}=-\left|z_{n}\right|$ is determined by the chosen angular aperture $\theta$ and the probe width $\mathrm{D}$ as [2]:

$$
\left|z_{n}\right|=\frac{D / 2}{\tan (\theta)}
$$

The transmit delays associated with the virtual source position are then computed as:

$$
\operatorname{delay}(x)=\left(\sqrt{x^{2}+z_{n}^{2}}-\left|z_{n}\right|\right) / c
$$

where $c$ is the speed of sound which value is classically taken as $1540 \mathrm{~m} \cdot \mathrm{s}^{-1}$ (sound propagation in soft tissue).

In this context, the traveling time of a diverging wave to reach a point scatterer positioned at $\left(x_{s}, z_{s}\right)$ in the medium

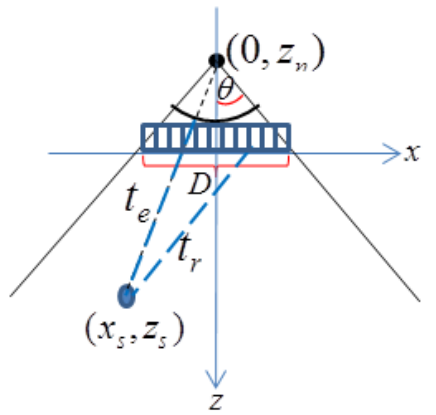

(a)

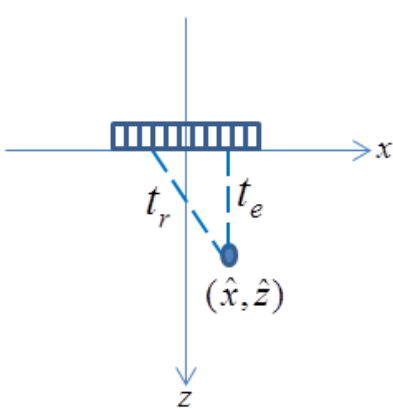

(b)
Fig. 2. Illustration of the time traveling concept (represented by $t_{e}$ and $t_{r}$ ) involved when either a diverging wave (a) or a plane wave (b) is used in emission. In both case, $\left(t_{e}+t_{r}\right)$ corresponds to the time needed by the insonifying wave to reach a scatterer (blue dot) and then come back to a specific transducer element. and to come back to a transducer element placed at $(x, 0)$ is given by:

$$
\tau_{d}(x)=\left(\sqrt{x_{s}^{2}+\left(z_{s}-z_{n}\right)^{2}}+z_{n}+\sqrt{\left(x_{s}-x\right)^{2}+z_{s}^{2}}\right) / c
$$

where $\sqrt{x_{s}^{2}+\left(z_{s}-z_{n}\right)^{2}}+z_{n}$ corresponds to the distance that the wave travels to reach the scatterer, and $\sqrt{\left(x_{s}-x\right)^{2}+z_{s}^{2}}$ corresponds to the distance between the scatterer point and the transducer element.

In the case of plane wave, the traveling time involved to reach a point scatterer $(\hat{x}, \hat{z})$ and to come back to the same transducer element $(x, 0)$ is given by:

$$
\tau_{p}(x)=\left(\hat{z}+\sqrt{(\hat{x}-x)^{2}+\hat{z}^{2}}\right) / c
$$

By making equal the value and first order derivative of the Taylor expansions of (3) and (4) at $x=0$, the following relation between $\left(x_{s}, z_{s}\right)$ and $(\hat{x}, \hat{z})$ can be derived:

$$
\left\{\begin{array}{l}
\hat{x}=\frac{x_{s}\left(\sqrt{x_{s}^{2}+\left(z_{s}-z_{n}\right)^{2}}+z_{n}+\sqrt{x_{s}^{2}+z_{s}^{2}}\right)}{z_{s}+\sqrt{x_{s}^{2}+z_{s}^{2}}} \\
\hat{z}=\frac{z_{s}\left(\sqrt{x_{s}^{2}+\left(z_{s}-z_{n}\right)^{2}}+z_{n}+\sqrt{x_{s}^{2}+z_{s}^{2}}\right)}{z_{s}+\sqrt{x_{s}^{2}+z_{s}^{2}}}
\end{array}\right.
$$

and

$$
\left\{\begin{array}{l}
x_{s}=\frac{\hat{x}\left(z_{n}^{2}-\left(\hat{z}+\sqrt{\hat{x}^{2}+\hat{z}^{2}}-z_{n}\right)^{2}\right)}{2\left(z_{n} \hat{z}-\left(\hat{z}+\sqrt{\hat{x}^{2}+\hat{z}^{2}}-z_{n}\right) \sqrt{\hat{x}^{2}+\hat{z}^{2}}\right)} \\
z_{s}=\frac{\hat{z}\left(z_{n}^{2}-\left(\hat{z}+\sqrt{\hat{x}^{2}+\hat{z}^{2}}-z_{n}\right)^{2}\right)}{2\left(z_{n} \hat{z}-\left(\hat{z}+\sqrt{\hat{x}^{2}+\hat{z}^{2}}-z_{n}\right) \sqrt{\hat{x}^{2}+\hat{z}^{2}}\right)}
\end{array}\right.
$$

From equations (5) and (6) the following generic algorithm is thus proposed to reconstruct a sectorial image: 1) emission of a diverging wave using a standard phased-array; 2) from the received echo, application of the UFSI technique derived for plane-wave. Thanks to equation (5), one can see that the output of this step will reconstruct the desired ultrasound image but expressed in the $(\hat{x}, \hat{z})$ coordinate system; 3$)$ application of the spatial transform given through equation (6) allows to express the reconstructed image in the conventional cartesian space.

It is worth pointing out that equations (5) and (6) have been obtained for the particular case where $x=0$, meaning that the equivalence between the traveling time of equation (3) and (4) is exact only at the center of the probe. We thus investigate the error in terms of traveling time difference when the relation (5) and (6) are used for each point in the medium. More precisely, for each point $\left(x_{s}, z_{s}\right)$ of the medium, equation (5) allows derivating the corresponding $(\hat{x}, \hat{z})$ point. For this new point, we then compute the time traveling difference for $x=0$ with the other elements of the probe and display the maximum value error in Fig.3 (the black line on the top models the transducer location). From Fig.3, one can observe that the maximum error appears in the near field with a value around $10^{-7}$ seconds. Moreover, as expected, there is no error at the middle of the probe and this error increases by approaching the border of the image. However, it is worth noting that the error remains lower than $5 \times 10^{-7}$ seconds on the entire medium, showing the marginal effect of such approximation. 


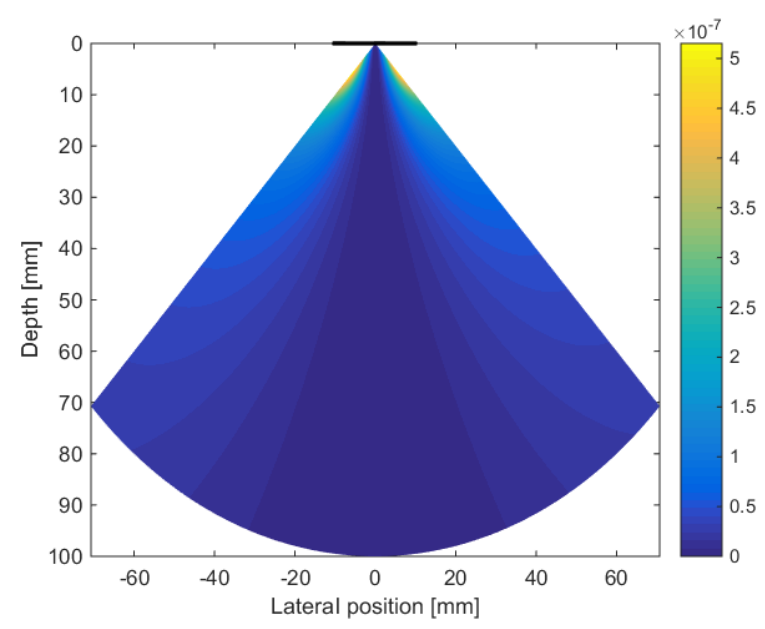

Fig. 3. Traveling time errors induced to space transformation.

\section{RESUlts}

The performance of the proposed scheme to reconstruct sectorial ultrasound images has been evaluated from both simulation and experimental data acquired using a Verasonics system and a standard phase array (Verasonics, P4-2). The corresponding probe settings are provided in TableI. The same properties of the probe were used for the simulation. All the presented results were compared with the method of Papadacci et al. [2]. Indeed, this method is currently considered as the reference in ultrafast imaging for sectorial acquisition. Contrary to the proposed approach, this technique corresponds to a spatial-based approach where the desired image is obtained from a delay-and-sum (DAS) beamforming.

TABLE I

PROBE SETTINGS

\begin{tabular}{ll}
\hline Parameters & Value \\
\hline Transmit Frequency & $2.5 \mathrm{MHz}$ \\
Sampling Frequency & $10 \mathrm{MHz}$ \\
Number of elements & 64 \\
Pitch & $0.32 \mathrm{~mm}$ \\
Kerf & $0.05 \mathrm{~mm}$ \\
Height & $13 \mathrm{~mm}$ \\
Elevation Focus & $60 \mathrm{~mm}$ \\
Apodization & None \\
\hline
\end{tabular}

\section{A. Simulations}

The correctness of the proposed approach has been first investigated through simulation using Field II software [4]. For each simulation, a single diverging wave is generated in emission, with a full-aperture consisting of 64 active elements and a $90^{\circ}$ angular aperture (the virtual source point being positioned at $(0,-D / 2)$, where $\mathrm{D}$ corresponds to the probe width).

First, the ability of the proposed method in reconstructing sectorial image has been investigated through the use of a simple numerical phantom composed of a set of discrete point scatterers. Fig. 4 presents the obtained results using the proposed method (Fig. 4a) and the one of Papadaci et

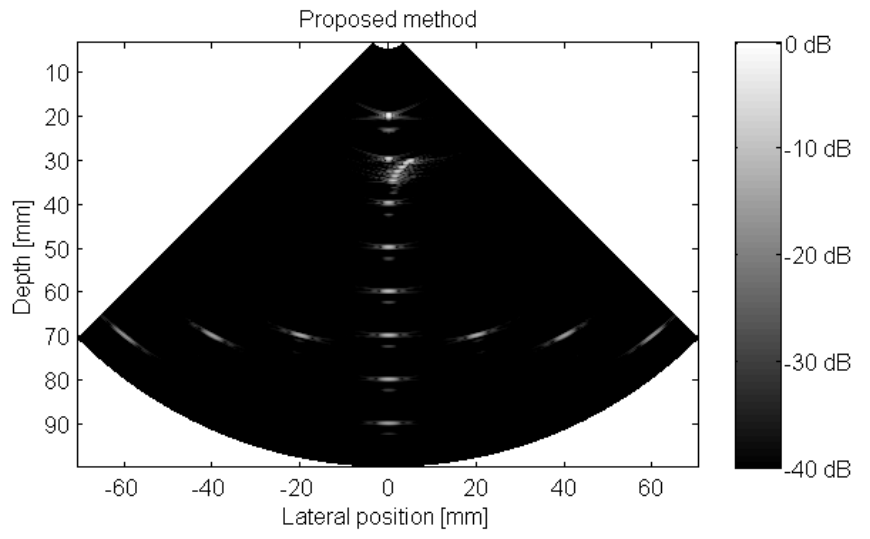

(a)

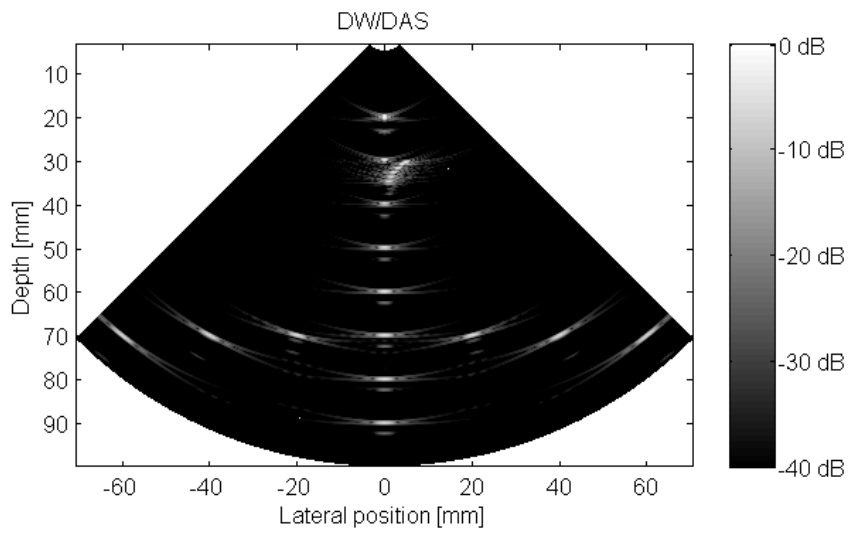

(b)

Fig. 4. Reconstructed point scatterers phantom Bmode image by (a) Proposed method and (b) DW/DAS.

al. (Fig. 4b). This result illustrates the capacity of our approach in obtaining competitive results with respect to the DW/DAS method.

The quality of the reconstructed images was then evaluated in simulation through the measurement of the classical contrast to noise ratio (CNR equation (17) in [5]). To this end, several simulated $1 \mathrm{~cm}$-diameter anechoic phantom embedded in a medium with high density of scatterers (20 per unit of resolution) were generated at different depths and for different lateral positions. For each position, the corresponding CNR has been computed for the proposed method and DW/DAS. Each given value provided in Fig. 5 corresponds to a mean and standard deviation measurement computed from 50 phantoms, each of them being generated using a distinct random scatterers set. From these figures, one can observe that the two algorithms exhibit similar performances in both directions, whereas our approach obtains slightly higher CNR scores. This aspect is further reinforced by the better quality of the reconstructed PSFs using our method (Fig. 4).

\section{B. In vitro experiments}

The proposed method was also evaluated experimentally using a CIRS phantom (model 054GS). After insonifying 


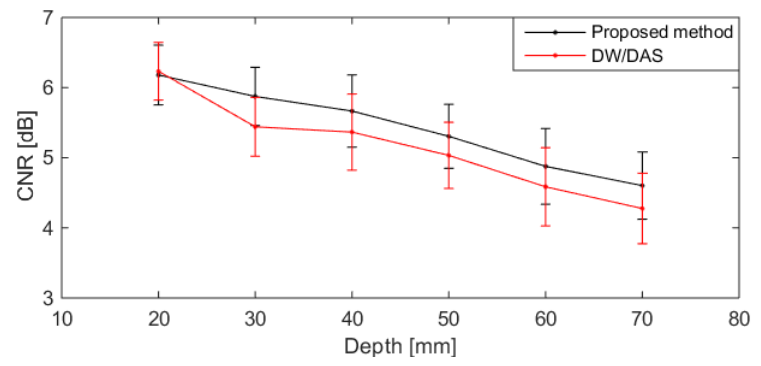

(a)

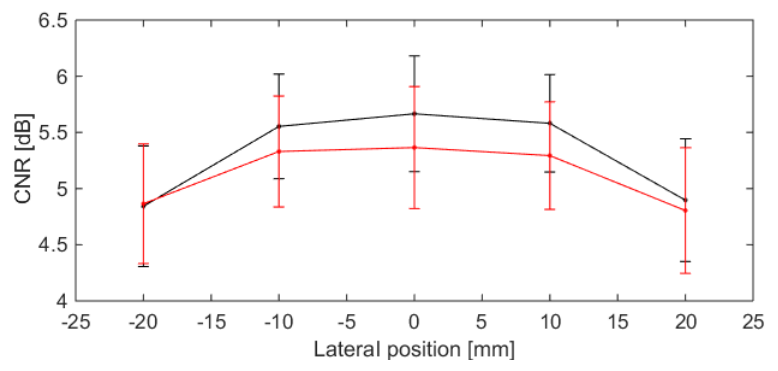

(b)

Fig. 5. Mean and standard deviation of CNR measurements as a function of (a) depth (b) lateral position (at $40 \mathrm{~mm}$ depth).

the phantom using a diverging wave, the received signals were then processed using either the algorithm proposed in this work or the reference DW/DAS method. Fig. 6 shows the B-mode image of CIRS phantom reconstructed by each methods. From this figure, one can observe that the image quality of the proposed method is comparable with the one obtained with DW/DAS. Since only one diverging wave is used, the image contrast decreases a lot with depth. This result shows the feasibility of reconstructing a sectorial image with one diverging wave insonification and the necessity of doing coherent diverging wave compounding to improve the image quality as in [2].

\section{CONCLUSIONS}

In this paper, the concept of UFSI was extended to reconstruct sectorial image using diverging wave in emission. The capacity of the proposed algorithm has been investigated on both simulation and experimental data. Results show that UFSI for sectorial imaging produces competitive results compared to the current reference DW/DAS method.

\section{ACKNOWLEDGMENT}

The RF Verasonics generator was cofounded by the FEDER program, Saint-Etienne Metropole (SME) and Conseil General de la Loire (CG42) within the framework of the SonoCardioProtection Project leaded by Pr Pierre Croisille and Dr Magalie Viallon as principal investigators. This work was performed within the framework of the LABEX PRIMES (ANR- 11LABX-0063) of Université de Lyon, within the program "Investissements d'Avenir" (ANR-11-IDEX-0007) operated by the French National Research Agency (ANR). This work was

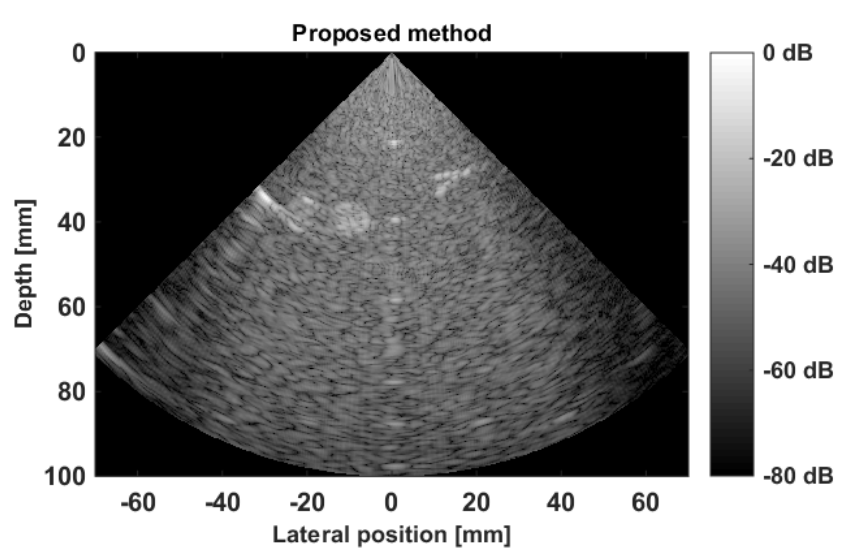

(a)

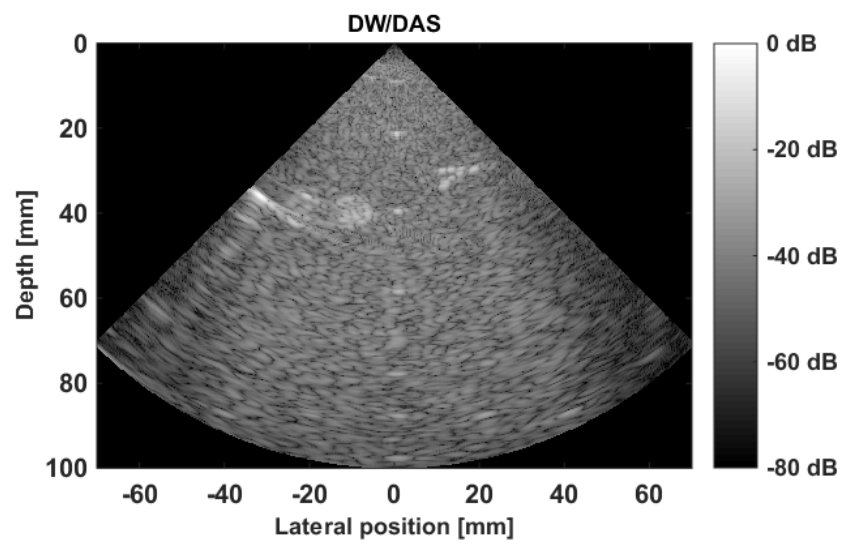

(b)

Fig. 6. Reconstructed Bmode image of CIRS phantom by (a) Proposed method (b) DW/DAS

also supported in part by the UltrasoundToGo RTD project (no. 20NA21 145911), evaluated by the Swiss NSF and funded by Nano-Tera.ch with Swiss Confederation financing.

\section{REFERENCES}

[1] H. Hasegawa and H. Kanai, "High-frame-rate echocardiography using diverging transmit beams and parallel receive beamforming," Journal of Medical Ultrasonics, vol. 38, no. 3, pp. 129-140, 2011.

[2] C. Papadacci, M. Pernot, M. Couade, M. Fink, and M. Tanter, "Highcontrast ultrafast imaging of the heart," Ultrasonics, Ferroelectrics and Frequency Control, IEEE Transactions on, vol. 61, no. 2, pp. 288-301, Feb 2014.

[3] O. Bernard, M. Zhang, F. Varray, P. Gueth, J.-P. Thiran, H. Liebgott, and D. Friboulet, "Ultrasound fourier slice imaging: a novel approach for ultrafast imaging technique," in Ultrasonics Symposium (IUS), 2014 IEEE International. Ieee, 2014, pp. 129-132.

[4] J. A. Jensen and N. B. Svendsen, "Calculation of pressure fields from arbitrarily shaped, apodized, and excited ultrasound transducers," Ultrasonics, Ferroelectrics, and Frequency Control, IEEE Transactions on, vol. 39, no. 2, pp. 262-267, 1992.

[5] D. Garcia, L. Tarnec, S. Muth, E. Montagnon, J. Porée, and G. Cloutier, "Stolt's f-k migration for plane wave ultrasound imaging," Ultrasonics, Ferroelectrics and Frequency Control, IEEE Transactions on, vol. 60, no. 9, pp. 1853-1867, Sep 2013. 